\title{
Regularity of the attractor for strongly damped wave equations with nonlinearity
}

Hongyan Li

\section{"Correspondence:}

lihongyan580@sohu.com

College of Management, Shanghai

University of Engineering Science,

Shanghai, 201620, China

\begin{abstract}
The long time behavior of the solutions for the strongly damped wave equation is considered with nonlinear damping, a nonlinear forcing term, and with a periodic boundary condition. We prove that the global attractor which captures all trajectories in $H^{1}(\Omega) \times L^{2}(\Omega)$ is a compact set in $H^{2}(\Omega) \times H^{1}(\Omega)$.
\end{abstract}

MSC: 35B40; 34A35; 37L30

Keywords: global attractor; strongly damped wave equation; compact set

\section{Introduction}

For $u(x, t): \Omega \times \mathbb{R}_{+} \rightarrow \mathbb{R}$, we consider the strongly damped wave equation on a bounded domain $\Omega$ in $\mathbb{R}^{3}$ with smooth boundary:

$$
u_{t t}-\alpha \Delta u_{t}-\Delta u+h\left(u_{t}\right)+f(u)=g(x),
$$

supplemented with the periodic boundary conditions and initial conditions

$$
u(0, x)=u_{0}(x), \quad u_{t}(0, x)=u_{1}(x), \quad x \in \Omega,
$$

where the strongly damped coefficient $\alpha$ is a positive constant, the damped function $h$ : $\mathbb{R} \rightarrow \mathbb{R}$ is continuous, and the forcing term $f: \mathbb{R} \rightarrow \mathbb{R}$ is a nonlinear term satisfying some growth conditions, $g: \Omega \rightarrow \mathbb{R}$ is the external force.

When $\alpha=0,(1.1)$ reduces to a usual wave equation with nonlinear damping, which arises as an evolutionary mathematical model in various systems ( $c f$. [1, 2]), for example: (i) modeling a continuous Josephson junction with specific $h, g$ and $f$; (ii) modeling a hybrid system of nonlinear waves and nerve conduct; and (iii) modeling a phenomenon in quantum mechanics and which has been studied widely by using of the concept of global attractors; see, for example, [2-10] for the linear damping case, and [11-18] for the nonlinear damping case. There are some results on the regularity in these papers; for example, in [7] the authors gave the dimensionality and related properties of the global attractor, and in [14], the authors presented a direct method to establish the optimal regularity of the attractor for the semilinear damped wave equation (when $\alpha=0$ and $h\left(u_{t}\right)=u_{t}$ ) with nonlinearity for the critical growth. But for $\alpha \neq 0$, the case can be complex.

There is a large literature on the asymptotic behavior of solutions to (1.1), (1.2) (cf. [1, 19-23]). For the strongly damped wave equations, [23] had proved the uniform bounded- 
ness of the global attractor for very strong damping in $H^{1} \times L^{2}$ and obtained an estimate of the upper bound of the Hausdorff dimension of an attractor for the strongly damped nonlinear wave equation (1.1). Furthermore, when $h\left(u_{t}\right)=0$, Pata and Zelik in [21] had proved the existence of compact global attractors of optimal regularity, i.e., the compact global attractor on $H^{1}(\Omega) \times L^{2}(\Omega)$ is a bounded subset of $H^{2} \times H^{1}$. The aim of this paper is to prove that the dynamical system associated with (1.1) possesses a compact global attractor in $H^{2}(\Omega) \times H^{1}(\Omega)$, i.e., we will prove that the global attractor $\mathcal{A}_{1}$ in $H^{1}(\Omega) \times L^{2}(\Omega)$ is a compact set $\mathcal{A}_{2}$ in $H^{2}(\Omega) \times H^{1}(\Omega)$. This implies that $\mathcal{A}_{1}=\mathcal{A}_{2}$.

The paper is organized as follows. In Section 2, we present the existence, uniqueness, and continuous dependence of solutions for problem (1.1), and we establish the existence of an absorbing set in $H^{1}(\Omega) \times L^{2}(\Omega)$. In Section 3, we prove the existence of the global attractor in $H^{1}(\Omega) \times L^{2}(\Omega)$. In Section 4, we establish the regularity of the attractor, i.e., that the global attractor is a compact subset in $H^{2}(\Omega) \times H^{1}(\Omega)$.

\section{Preliminaries}

We assume that $g \in L^{2}(\Omega)=H$, and $\dot{H}^{-1}=\left(\dot{H}^{1}\right)^{*}$, with

$$
\dot{H}^{1}=H^{1} \cap\left\{u \in L^{2}(\Omega) ; \int_{\Omega} u(x) d x=0\right\},
$$

and the functions $h, f$ satisfy the following conditions:

(i) Let $f(u) \in C^{1}(\mathbb{R} ; \mathbb{R})$ satisfy:

(1) The asymptotic sign condition

$$
\limsup _{|s| \rightarrow+\infty} \frac{f(s)}{s} \leq 0
$$

(2) Let $F(s)=\int_{0}^{s} f(\rho) d \rho$, there exist constants $c_{1}, c_{2}, \delta, C_{\delta}>0$ such that

$$
(f(u), u)-c_{1} \int_{\Omega} F(u) d x-\delta|u|^{2} \geq-C_{\delta}|\Omega|
$$

where $|\cdot|_{0}$ denotes the absolute value of the number in $\mathbb{R}$. We have

$$
\left|f^{\prime}(s)\right| \leq c_{2}\left(1+|s|^{p}\right) \quad \text { with } \begin{cases}0 \leq p<\infty, & \text { when } n=1,2, \\ 0 \leq p<2, & \text { when } n=3\end{cases}
$$

(ii) There exist two constants $\beta_{1}^{\prime}, \beta_{2}^{\prime} \geq 0$ such that

$$
h(0)=0, \quad 0<\beta_{1}^{\prime} \leq h^{\prime}(s) \leq \beta_{2}^{\prime}<+\infty, \quad \forall s \in \mathbb{R} .
$$

Let $A u=-\Delta u$, then the system (1.1)-(1.2) is equivalent to the following initial value problem in $\dot{H}^{1}(\Omega) \times L^{2}(\Omega)$ :

$$
\left\{\begin{array}{l}
\dot{Y}=P Y+Q(Y), \quad x \in \Omega, t>0, \\
Y(0)=Y_{0}=\left(u_{0}, u_{1}\right)^{T} \in \dot{H} \times L^{2},
\end{array}\right.
$$


where $Y=\left(u, u_{t}\right)^{T}, Q(Y)=\left(0,-h\left(u_{t}\right)-f(u)+g\right)^{T}$,

$$
P=\left(\begin{array}{cc}
0 & I \\
-A & -k A
\end{array}\right)
$$

By the assumption (2.1)-(2.4), it is easy to check that the function $Q(Y): \dot{H}^{1} \times L^{2} \rightarrow \dot{H}^{1} \times$ $L^{2}$ is continuously differentiable and globally Lipschitz continuous with respect to $Y$. By the classical theory concerning the existence and uniqueness of the solutions of evolution differential equations ( $c f$. [15]), we have the following lemma (see [23] for details).

Lemma 2.1 Consider the initial value problem (2.5) in $\dot{H}^{1} \times L^{2}$. If (2.1)-(2.4) hold, then, for any $Y_{0} \in \dot{H}^{1} \times L^{2}$, there exists a unique continuous function $Y(\cdot)=Y\left(\cdot, Y_{0}\right) \in C\left(\mathbb{R}_{+} ; \dot{H}^{1} \times L^{2}\right)$ such that $Y\left(0, Y_{0}\right)=Y_{0}$ and $Y(t)$ satisfies the integral equation

$$
Y\left(t, Y_{0}\right)=e^{P t} Y_{0}+\int_{0}^{t} e^{P(t-\tau)} Q(Y(\tau)) d \tau
$$

$Y\left(t, Y_{0}\right)$ is called the mild solution of $(2.5)$, and $Y\left(t, Y_{0}\right)$ is jointly continuous in $t$ and $Y_{0}$, and

$$
\left(u, u_{t}\right) \in C\left(\mathbb{R}_{+} ; \dot{H}^{1}(\Omega)\right) \times\left[C\left(\mathbb{R}_{+} ; L^{2}(\Omega)\right) \cap L^{2}\left(\left(0, T^{*}\right) ; \dot{H}^{1}(\Omega)\right)\right], \quad \forall T^{*}>0
$$

For any $t \geq 0$, we can introduce a map

$$
S(t): Y_{0}=\left(u_{0}, u_{1}\right) \rightarrow\left(u, u_{t}\right)=Y\left(t, Y_{0}\right), \quad S(t): \dot{H}^{1} \times L^{2} \rightarrow \dot{H}^{1} \times L^{2}
$$

where $Y\left(t, Y_{0}\right)$ is the solution of (2.5), and then $\{S(t) \mid t \geq 0\}$ is a continuous semigroup on $\dot{H}^{1} \times L^{2}$.

Consider the map $G: \dot{H}^{1} \times L^{2} \rightarrow \dot{H}^{1} \times L^{2}$ defined as

$$
G\left(u(t), u_{t}(t)\right)=e^{P t}\left(u_{0}, u_{1}\right)+\int_{0}^{t} e^{P(t-\tau)}\left(0, g-h\left(u_{t}(\tau)\right)-f(u(\tau))\right) d \tau \text {. }
$$

For some $\lambda>0$, let

$$
A_{1}=A+\lambda I
$$

Let

$$
\begin{aligned}
& (u, v)=\int_{\Omega} u v d x, \quad|u|=(u, u)^{\frac{1}{2}}, \quad \forall u, v \in L^{2}, \\
& ((u, v))=\int_{\Omega} A_{1} u \cdot v d x, \quad\|u\|=((u, u))^{\frac{1}{2}}, \quad \forall u, v \in H^{1}(\Omega) .
\end{aligned}
$$

Obviously, $A_{1}$ is symmetric and positive definite, so we have the following Poincaré type inequality:

$$
\|y\| \geq \sqrt{\lambda}|y|, \quad y \in L^{2}
$$


Let $h_{1}\left(u_{t}\right)=h\left(u_{t}\right)-\alpha \lambda u_{t}$, then (1.1) can be written in the equivalent form

$$
u_{t t}+\alpha A_{1} u_{t}+A_{1} u+h_{1}\left(u_{t}\right)-\lambda u+f(u)=g
$$

and by $(2.4), h_{1}\left(u_{t}\right)$ satisfies

$$
h_{1}(0)=0, \quad-\alpha \lambda<\beta_{1} \leq h_{1}^{\prime}(\theta) \leq \beta_{2}<+\infty, \quad \forall \theta \in \mathbb{R},
$$

where $\beta_{1}=\beta_{1}^{\prime}-\alpha \lambda, \beta_{2}=\beta_{2}^{\prime}-\alpha \lambda$ are two constants.

To construct an attractor for (1.1), we make the following assumptions. Let $\varphi=(u, v)^{T}$, $v=u_{t}+k u$, where $k$ is chosen as

$$
k=\frac{\alpha \lambda+\beta_{1}}{4+2\left(\alpha \lambda+\beta_{1}\right) \alpha+\beta_{2}^{2} / \lambda_{1}} .
$$

Equation (2.9) can be written as

$$
\varphi_{t}+H(\varphi)=F(\varphi), \quad \varphi(0)=\left(u_{0}, v_{0}=u_{1}+k u_{0}\right)^{T}, \quad t \geq 0,
$$

where

$$
\begin{aligned}
& F(\varphi)=\left(\begin{array}{c}
0 \\
\lambda u-f(u)+g
\end{array}\right), \\
& H(\varphi)=\left(\begin{array}{c}
k u-v \\
A_{1} u-k\left(\alpha A_{1}-k\right) u+\left(\alpha A_{1}-k\right) v
\end{array}\right)+\left(\begin{array}{c}
0 \\
h_{1}(v-k u)
\end{array}\right) .
\end{aligned}
$$

We define a new weighted inner product and norm in $E=\dot{H}^{1} \times L^{2}$ as

$$
(\varphi, \psi)_{E}=\mu\left(\left(u_{1}, u_{2}\right)\right)+\left(v_{1}, v_{2}\right), \quad\|\varphi\|_{E}=(\varphi, \varphi)_{E}^{1 / 2},
$$

for any $\varphi=\left(u_{1}, v_{1}\right)^{T}, \psi=\left(u_{2}, v_{2}\right)^{T} \in E$, where $\mu$ is chosen as

$$
\mu=\frac{4+\left(\alpha \lambda+\beta_{1}\right) \alpha+\beta_{2}^{2} / \lambda}{4+2\left(\alpha \lambda+\beta_{1}\right) \alpha+\beta_{2}^{2} / \lambda} \in\left(\frac{1}{2}, 1\right) .
$$

Obviously, the norm $\|\cdot\|_{E}$ in (2.13) is equivalent to the usual norm $|\cdot|_{\dot{H}^{1} \times L^{2}}$ in $E$.

Lemma 2.2 (see [23], Lemma 1) For any $\varphi=(u, v)^{T} \in E$, if

$$
-\alpha \lambda<\beta_{1} \leq \beta_{2}<+\infty, \quad \beta_{2} \geq\left|\beta_{1}\right|+\min \left\{1 / \alpha,\left(\alpha \lambda+\beta_{1}\right) / 2\right\},
$$

hold, then

$$
(H(\varphi), \varphi)_{E} \geq \sigma\|\varphi\|_{E}^{2}+\frac{\alpha}{2}\|v\|^{2}+\frac{\beta_{1}}{2}|v|^{2} \geq \sigma\|\varphi\|_{E}^{2}+\frac{\alpha \lambda+\beta_{1}}{2}|v|^{2},
$$

where

$$
\sigma=\frac{\alpha \lambda+\beta_{1}}{\gamma_{1}+\sqrt{\gamma_{1} \gamma_{2}}}, \quad \gamma_{1}=4+\left(\alpha \lambda+\beta_{1}\right) \alpha+\frac{\beta_{2}^{2}}{\lambda}, \quad \gamma_{2}=\left(\alpha \lambda+\beta_{1}\right) \alpha+\frac{\beta_{2}^{2}}{\lambda} .
$$


Proposition 2.1 The semigroup $\{S(t) \mid t \geq 0\}$ possesses an absorbing set $\mathcal{B} \subset \dot{H}^{1} \times L^{2}$.

Proof Let $\varphi=(u, v)^{T} \in E$ be the solution of (2.12). Taking the inner product $(\cdot, \cdot)_{E}$ of $(2.12)$ with $\varphi$, we have

$$
\left(\varphi_{t}, \varphi\right)+(H(\varphi), \varphi)=(F(\varphi), \varphi)
$$

and

$$
\frac{1}{2} \frac{d}{d t}\|\varphi\|_{E}^{2}+(H(\varphi), \varphi)_{E}+(f(u), v)-\lambda(u, v)=(g, v) .
$$

By $v=u_{t}+k u$, we have

$$
(f(u), v)=\left(f(u), u_{t}+k u\right)=\frac{d}{d t} \int_{\Omega} F(u) d x+k(f(u), u),
$$

by (2.18) and (2.19), we have

$$
\frac{1}{2} \frac{d}{d t}\left[\|\varphi\|_{E}^{2}+2 \int_{\Omega} F(u) d x\right]+(H(\varphi), \varphi)_{E}+k(f(u), u)-\lambda(u, v)=(g, v) .
$$

Let $y(t)=\|\varphi\|_{E}^{2}+2 \int_{\Omega} F(u) d x+2 C_{\delta}|\Omega| \geq \frac{1}{4}\|\varphi\|_{E}^{2} \geq 0$. By (2.15) and (2.2), there exists $\delta>0$ such that

$$
\begin{aligned}
(H(\varphi), \varphi)_{E}+k(f(u), u)-\lambda(u, v) \\
\geq \sigma\|\varphi\|_{E}^{2}+\frac{\alpha \lambda+\beta_{1}}{2}|v|^{2}+k c_{1} \int_{\Omega} F(u) d x+k \delta|u|^{2}-k C_{\delta}|\Omega|-\lambda|u||v| \\
\geq \sigma\|\varphi\|_{E}^{2}+\frac{\alpha \lambda+\beta_{1}}{2}|v|^{2}+k c_{1} \int_{\Omega} F(u) d x \\
\quad+\frac{k \delta}{\lambda \mu} \mu|u|^{2}-k C_{\delta}|\Omega|-\frac{\sqrt{\lambda}}{2 \sqrt{\mu}}\|\varphi\|_{E}^{2} \\
\geq\left(\sigma+\frac{k \delta}{\lambda \mu}-\frac{\sqrt{\lambda}}{2 \sqrt{\mu}}\right)\|\varphi\|_{E}^{2}+k c_{1} \int_{\Omega} F(u) d x-k C_{\delta}|\Omega|+\frac{\alpha \lambda+\beta_{1}}{2}|v|^{2} \\
\geq \frac{1}{2} \rho y-k\left(C_{\delta}|\Omega|+c_{1} C_{\delta}|\Omega|\right)+\frac{\alpha \lambda+\beta_{1}}{2}|v|^{2},
\end{aligned}
$$

where $\rho=\min \left\{k c_{1},\left(\sigma+\frac{k \delta}{\lambda \mu}-\frac{\sqrt{\lambda}}{2 \sqrt{\mu}}\right)\right\}$. By $(2.20)$ and $(2.21)$,

$$
\begin{aligned}
\frac{d}{d t} y+\rho y & \leq 2 k C_{\delta}|\Omega|\left(1+c_{1}\right)+2(g, v)-\left(\alpha \lambda+\beta_{1}\right)|v|^{2} \\
& \leq 2 k C_{\delta}|\Omega|\left(1+c_{1}\right)+\frac{1}{\alpha \lambda+\beta_{1}}|g|^{2} .
\end{aligned}
$$

Using the Gronwall lemma, we have

$$
\|\varphi(t)\|_{E}^{2} \leq 4 y(0) e^{-2 \rho t}+4\left(\frac{|g|^{2}}{\left(\alpha \lambda+\beta_{1}\right) \rho}+\frac{2 k C_{\delta}|\Omega|\left(1+c_{1}\right)}{\rho}\right) .
$$


Following [24] and [8], it follows from (2.1) that, for each $\kappa>0$, there is a constant $C_{\kappa}>0$ such that, for each $u \in L^{2}(\Omega)$,

$$
(f(u), u)=\int_{\Omega} f(u) u d x \leq \kappa|u|^{2}+C_{\kappa} .
$$

Note that (2.2) and (2.23) imply

$$
\begin{aligned}
c_{1} \int_{\Omega} F(u) d x & \leq(f(u), u)-\delta|u|^{2}+C_{\delta}|\Omega| \\
& \leq \kappa|u|^{2}+C_{\kappa}-\delta|u|^{2}+C_{\delta}|\Omega| \leq c\left(1+|u|^{2}\right) .
\end{aligned}
$$

For any bounded set $B$ of $E$, where $\sup _{\varphi \in B}\|\varphi\|_{E}<r$, if $\varphi(0) \in B$, there exists $c_{2}=c_{2}(r)>0$ such that $y(0)=\|\varphi(0)\|_{E}^{2}+2 \int_{\Omega} F\left(u_{0}\right) d x+2 C_{\delta}|\Omega| \leq c_{2}$. Therefore, for the solution $\varphi(t)=$ $(u(t), v(t))^{T}$ of $(2.12)$ with $\varphi(0) \in B$,

$$
\|\varphi(t)\|_{E}^{2} \leq 4 c_{2}(r) e^{-2 \rho t}+4\left(\frac{|g|^{2}}{\left(\alpha \lambda+\beta_{1}\right) \rho}+\frac{2 k C_{\delta}|\Omega|\left(1+c_{1}\right)}{\rho}\right) .
$$

Taking

$$
M_{0}=8\left(\frac{|g|^{2}}{\left(\alpha \lambda+\beta_{1}\right) \rho}+\frac{2 k C_{\delta}|\Omega|\left(1+c_{1}\right)}{\rho}\right)
$$

completes the proof.

\section{Existence of the global attractor in $\dot{H}^{1} \times L^{2}$}

Theorem 3.1 (see [2, I.1.1]) Let $\{S(t) \mid t \geq 0\}$ be a continuous semigroup on $\dot{H}^{1} \times L^{2}$ that possesses an absorbing ball in $\dot{H}^{1} \times L^{2}$. Let us assume that, for any $t \geq 0$,

$$
S(t)=S_{1}(t)+S_{2}(t)
$$

where:

- For every bounded set $B$, there exists $t_{0}$ that depends on $B$, such that $\bigcup_{t \geq t_{0}} S_{1}(t) B$ is relatively compact in $\dot{H}^{1} \times L^{2}$.

- For every bounded set $B$,

$$
\limsup _{t \rightarrow \infty}\left(\sup _{\theta \in B}\left\|S_{2}(t) \theta\right\|_{\dot{H}^{1} \times L^{2}}\right)=0 \text {. }
$$

Then $S(t)_{t \geq 0}$ possesses a global attractor $\mathcal{A}$ that is compact in $\dot{H}^{1} \times L^{2}$.

Remark 3.1 (see [10]) We characterize such an attractor $\mathcal{A}$ as

$$
\begin{aligned}
\mathcal{A}= & \left\{\left(u_{0}, u_{1}\right) \in \dot{H}^{1} \times L^{2},\left(u_{0}^{n}, u_{1}^{n}\right)_{n} \subset \mathcal{B}, \exists\left(t_{n}\right) \rightarrow \infty,\right. \\
& \text { such that } \left.\left\|S\left(t_{n}\right)\left(u_{0}^{n}, u_{1}^{n}\right)-\left(u_{0}, u_{1}\right)\right\|_{\dot{H}^{1} \times L^{2}} \rightarrow 0\right\} .
\end{aligned}
$$

Theorem 3.2 The semigroup $\{S(t) \mid t \geq 0\}$ possesses a global attractor $\mathcal{A}$ in $\dot{H}^{1} \times L^{2}$. 
Proof We consider $g_{\epsilon} \in C_{0}^{\infty}$ and $\int_{0}^{1} g_{\epsilon}(x) d x=0$ such that

$$
\left|g-g_{\epsilon}\right|_{L^{2}}<\epsilon
$$

and we introduce the splitting $(u, v)=\left(u_{1}, v_{1}\right)+\left(u_{2}, v_{2}\right)+\left(u_{3}, v_{3}\right)$ where $\left(u_{1}, v_{1}\right)$ satisfies

$$
\left\{\begin{array}{l}
u_{1, t}+k u_{1}-v_{1}=0, \\
v_{1, t}+A_{1} u_{1}-k\left(\alpha A_{1}-k\right) u_{1}+\left(\alpha A_{1}-k\right) v_{1}+h_{1}\left(v_{1}-k u_{1}\right)+f(u)-\lambda u_{1}=g_{\epsilon}, \\
u_{1}(0)=0, \quad v_{1}(0)=0,
\end{array}\right.
$$

$\left(u_{2}, v_{2}\right)$ satisfies

$$
\left\{\begin{array}{l}
u_{2, t}+k u_{2}-v_{2}=0, \\
v_{2, t}+A_{1} u_{2}-k\left(\alpha A_{1}-k\right) u_{2}+\left(\alpha A_{1}-k\right) v_{2} \\
\quad+h_{1}\left(v_{1}-k u_{1}+v_{2}-k u_{2}\right)-h_{1}\left(v_{1}-k u_{1}\right)+f\left(u_{1}+u_{2}\right)-f\left(u_{1}\right)-\lambda u_{2}=g-g_{\epsilon}, \\
u_{2}(0)=0, \quad v_{2}(0)=0,
\end{array}\right.
$$

and $\left(u_{3}, v_{3}\right)$ is the solution of

$$
\left\{\begin{array}{l}
u_{3, t}+k u_{3}-v_{3}=0 \\
v_{3, t}+A_{1} u_{3}-k\left(\alpha A_{1}-k\right) u_{3}+\left(\alpha A_{1}-k\right) v_{3} \\
\quad+h_{1}(v-k u)-h_{1}\left(v_{1}-k u_{1}+v_{2}-k u_{2}\right)+f(u)-f\left(u_{1}+u_{2}\right)-\lambda u_{3}=0 \\
u_{3}(0)=u_{0}, \quad v_{3}(0)=v_{0}
\end{array}\right.
$$

We now define the families of maps $\left\{S_{k}^{1}(t) \mid t \geq 0\right\}$ and $\left\{S_{k}^{2}(t) \mid t \geq 0\right\}$ in $\dot{H}^{1} \times L^{2}$, where

$$
S_{k}^{1}(t)\left(u_{0}, v_{0}\right)=\left(u_{1}(t), v_{1}(t)\right)+\left(u_{2}(t), v_{2}(t)\right), \quad S_{k}^{2}(t)\left(u_{0}, v_{0}\right)=\left(u_{3}(t), v_{3}(t)\right) .
$$

First step: We prove that $\left(u_{1}, v_{1}\right)$ is bounded in $H^{2} \times H^{1}$. The system (3.2) can be written as

$$
\varphi_{t}^{1}+H_{1}\left(\varphi^{1}\right)=F_{1}\left(\varphi^{1}\right), \quad \varphi^{1}(0)=(0,0)^{T}, \quad t \geq 0,
$$

where

$$
\begin{aligned}
& F_{1}\left(\varphi^{1}\right)=\left(\begin{array}{c}
0 \\
f\left(u_{1}\right)+\lambda u_{1}+g_{\epsilon}
\end{array}\right), \\
& H_{1}\left(\varphi^{1}\right)=\left(\begin{array}{c}
k u_{1}-v_{1} \\
A_{1} u_{1}-k\left(\alpha A_{1}-k\right) u_{1}+\left(\alpha A_{1}-k\right) \nu_{1}+h\left(v_{1}-k u_{1}\right)
\end{array}\right) .
\end{aligned}
$$

Similar to Proposition 2.1, we obtain

$$
\left\|\varphi^{1}\right\|_{E}^{2} \leq \frac{1}{\rho} C\left(\left|g_{\epsilon}\right|_{H^{-1}}, \lambda, \alpha, \delta, \kappa, k, \beta_{1}, \beta_{2}\right)=M_{1}
$$

Now we multiply (3.2) by $\left(A_{1} u_{1}, A_{1} v_{1}\right)$ and integrate on $\Omega$ to obtain

$$
\frac{1}{2} \frac{d}{d t}\left\|A_{1}^{\frac{1}{2}} \varphi^{1}\right\|_{E}^{2}+\sigma\left\|A_{1}^{\frac{1}{2}} \varphi^{1}\right\|_{E}^{2}+\frac{\alpha \lambda+\beta_{1}}{2}\left|A_{1}^{\frac{1}{2}} v_{1}\right|^{2} \leq\left(-f\left(u_{1}\right)+\lambda u_{1}, A_{1} v_{1}\right)+\left(g_{\epsilon}, A_{1} v_{1}\right)
$$


with

$$
\begin{aligned}
\left(-f\left(u_{1}\right), A_{1} v_{1}\right) & =\left(-A_{1}^{\frac{1}{2}} f\left(u_{1}\right), A_{1}^{\frac{1}{2}}\left(u_{1, t}+k u_{1}\right)\right) \\
& =-\frac{d}{d t}\left(A_{1}^{\frac{1}{2}} f(u), A_{1}^{\frac{1}{2}} u_{1}\right)+\left(A_{1}^{\frac{1}{2}} f^{\prime}\left(u_{1}\right) u_{1, t}, A_{1}^{\frac{1}{2}} u_{1}\right)-k\left(A_{1}^{\frac{1}{2}} f(u), A_{1}^{\frac{1}{2}} u_{1}\right) .
\end{aligned}
$$

Then (3.8) can be rewritten as

$$
\begin{aligned}
& \frac{1}{2} \frac{d}{d t}\left[\left\|A_{1}^{\frac{1}{2}} \varphi^{1}\right\|_{E}^{2}+2\left(A_{1}^{\frac{1}{2}} f\left(u_{1}\right), A_{1}^{\frac{1}{2}} u_{1}\right)\right]+\sigma\left\|A_{1}^{\frac{1}{2}} \varphi^{1}\right\|_{E}^{2}+k\left(A_{1}^{\frac{1}{2}} f\left(u_{1}\right), A_{1}^{\frac{1}{2}} u_{1}\right) \\
& \quad \leq-\frac{\alpha \lambda+\beta_{1}}{2}\left|A_{1}^{\frac{1}{2}} v_{1}\right|^{2}+\left(A_{1}^{\frac{1}{2}} f^{\prime}\left(u_{1}\right) u_{1, t}, A_{1}^{\frac{1}{2}} u_{1}\right)+\lambda\left(A_{1}^{\frac{1}{2}} u_{1}, A_{1}^{\frac{1}{2}} v_{1}\right)+\left(A_{1}^{\frac{1}{2}} g_{\epsilon}, A_{1}^{\frac{1}{2}} v_{1}\right),
\end{aligned}
$$

i.e.,

$$
\begin{aligned}
& \frac{1}{2} \frac{d}{d t}\left[\left\|A_{1}^{\frac{1}{2}} \varphi^{1}\right\|_{E}^{2}+2\left(A_{1}^{\frac{1}{2}} f\left(u_{1}\right), A_{1}^{\frac{1}{2}} u_{1}\right)\right]+\left(\sigma-\frac{\sqrt{\lambda}}{2 \sqrt{\mu}}\right)\left\|A_{1}^{\frac{1}{2}} \varphi^{1}\right\|_{E}^{2}+k\left(A_{1}^{\frac{1}{2}} f\left(u_{1}\right), A_{1}^{\frac{1}{2}} u_{1}\right) \\
& \quad \leq\left(A_{1}^{\frac{1}{2}} f^{\prime}\left(u_{1}\right) u_{1, t}, A_{1}^{\frac{1}{2}} u_{1}\right)+\frac{A_{1}^{\frac{1}{2}} g_{\epsilon}}{2\left(\alpha \lambda+\beta_{1}\right)}
\end{aligned}
$$

for the first term on the right-hand side of (3.9), we have

$$
\begin{aligned}
\left(A_{1}^{\frac{1}{2}} f^{\prime}\left(u_{1}\right) u_{1, t}, A_{1}^{\frac{1}{2}} u_{1}\right) & \leq c_{3}\left|A_{1}^{\frac{1}{2}} u_{1, t}\right|\left|u_{1}\right|_{6}^{p}\left|A_{1}^{\frac{1}{2}} u_{1}\right|_{\frac{6}{3-p}} \\
& \leq\left. c_{3}\left|A_{1}^{\frac{1}{2}} u_{1, t}\right|\left|u_{1}\right|_{H^{1}}^{p} A_{1}^{\frac{1}{2}} u_{1}\right|_{\frac{2-p}{2}}\left|A_{1}^{\frac{1}{2}} u_{1}\right|_{\frac{p}{2}} \\
& \leq c_{3} \lambda^{\frac{p-2}{4}}\left|A_{1}^{\frac{1}{2}} u_{1, t}\right|\left|u_{1}\right|_{H^{1}}^{p}\left|A_{1} u_{1}\right| \\
& \leq c_{4}\left|A_{1} u_{1}\right|^{2}+c_{4} \lambda^{\frac{p-4}{2}}\left|A_{1}^{\frac{1}{2}} u_{1, t}\right|^{2}\left|u_{1}\right|_{H^{1}}^{2 p} \\
& \leq c_{4}\left|A_{1} u_{1}\right|^{2}+c_{5} \lambda^{\frac{p-4}{2}}\left(1+\left|u_{1}\right|_{H^{1}}^{6}+\left|v_{1}\right|_{H^{1}}^{6}\right) \\
& \leq c_{6}|| A_{1}^{\frac{1}{2}} \varphi^{1} \|_{E}^{2}+c_{5} \lambda^{\frac{p-4}{2}}\left(1+\left|u_{1}\right|_{H^{1}}^{6}+\left|v_{1}\right|^{6}\right) .
\end{aligned}
$$

By (3.9)-(3.10), we have

$$
\begin{aligned}
& \frac{d}{d t}\left[\left\|A_{1}^{\frac{1}{2}} \varphi^{1}\right\|_{E}^{2}+2\left(A_{1}^{\frac{1}{2}} f\left(u_{1}\right), A_{1}^{\frac{1}{2}} u_{1}\right)\right] \\
& \quad+2\left(\sigma-\frac{\sqrt{\lambda}}{2 \sqrt{\mu}}-c_{6}\right)\left\|A_{1}^{\frac{1}{2}} \varphi^{1}\right\|_{E}^{2}+2 k\left(A_{1}^{\frac{1}{2}} f\left(u_{1}\right), A_{1}^{\frac{1}{2}} u_{1}\right) \\
& \quad \leq 2 c_{5} \lambda^{\frac{p-4}{2}}\left(\left|u_{1}\right|_{H^{1}}^{6}+\left|v_{1}\right|^{6}\right)+\frac{A_{1}^{\frac{1}{2}} g_{\epsilon}}{\alpha \lambda+\beta_{1}} .
\end{aligned}
$$

Let $\varrho=\min \left\{\sigma-\frac{\sqrt{\lambda}}{2 \sqrt{\mu}}-c_{6}, k\right\} \geq 0$, using the Gronwall lemma, we have

$$
\begin{aligned}
& \left\|A_{1}^{\frac{1}{2}} \varphi^{1}\right\|_{E}^{2}+2\left(A_{1}^{\frac{1}{2}} f\left(u_{1}\right), A_{1}^{\frac{1}{2}} u_{1}\right) \\
& \quad \leq\left(\left\|A_{1}^{\frac{1}{2}} \varphi^{1}(0)\right\|_{E}^{2}+2\left(A_{1}^{\frac{1}{2}} f\left(u_{1}(0)\right), A_{1}^{\frac{1}{2}} u_{1}(0)\right)\right) e^{-2 \varrho t}
\end{aligned}
$$




$$
\begin{aligned}
& +2 c_{5} \lambda^{\frac{p-4}{2}} \int_{0}^{t} e^{-2 \varrho t}\left(\left|u_{1}(s)\right|_{H^{1}}^{6}+\left|v_{1}(s)\right|^{6}\right) d s+\frac{\left|A_{1}^{\frac{1}{2}} g_{\epsilon}\right|}{\left(\alpha \lambda+\beta_{1}\right) \varrho} \\
\leq & 2 c_{5} \lambda^{\frac{p-4}{2}} \int_{0}^{t} e^{-2 \varrho t}\left(\left|u_{1}(s)\right|_{H^{1}}^{6}+\left|v_{1}(s)\right|^{6}\right) d s+\frac{\left|A_{1}^{\frac{1}{2}} g_{\epsilon}\right|}{\left(\alpha \lambda+\beta_{1}\right) \varrho} .
\end{aligned}
$$

By (3.7), we have

$$
\left\|A_{1}^{\frac{1}{2}} \varphi^{1}\right\|_{E}^{2}+2\left(A_{1}^{\frac{1}{2}} f\left(u_{1}\right), A_{1}^{\frac{1}{2}} u_{1}\right) \leq \frac{1}{\rho \varrho} C^{3}\left(\left|A_{1}^{\frac{1}{2}} g_{\epsilon}\right|, \lambda, \alpha, \delta, \kappa, k, \beta_{1}, \beta_{2}\right) .
$$

Note that (3.2) implies that

$$
\left(A_{1}^{\frac{1}{2}} f\left(u_{1}\right), A_{1}^{\frac{1}{2}} u_{1}\right) \leq c_{7}\left|A_{1}^{\frac{1}{2}} u_{1}\right|^{2}+c_{7}\left|A_{1}^{\frac{1}{2}} u_{1}\right| \leq c_{8}\left\|A_{1}^{\frac{1}{2}} \varphi^{1}\right\|_{E}^{2}
$$

Then we obtain

$$
\left\|A^{\frac{1}{2}} \varphi^{1}\right\|_{E}^{2} \leq \frac{1}{\rho \varrho} C^{3}\left(\left|A_{1}^{\frac{1}{2}} g_{\epsilon}\right|, \lambda, \alpha, \delta, \kappa, k, \beta_{1}, \beta_{2}\right) .
$$

Proposition 2.1, (3.8), and (3.12) imply that $\left(u_{1}, v_{1}\right)$ is bounded in $H^{2} \times H^{1}$.

Second step: Let $\varphi^{2}=\left(u_{2}, v_{2}\right)$; we will prove that there exists a $K>0$ independence on $\epsilon$ such that

$$
\left\|\varphi^{2}\right\|_{E} \leq K \epsilon
$$

Multiplying (3.3) by $\left(u_{2}, v_{2}\right)$, we thus obtain

$$
\begin{aligned}
& \frac{1}{2} \frac{d}{d t}\left\|\varphi^{2}\right\|_{E}^{2}+\left(\sigma-\frac{2 \sqrt{\lambda}}{\sqrt{\mu}}\right)\left\|\varphi^{2}\right\|_{E}^{2}+\frac{\alpha \lambda+\beta_{1}}{2}\left|v_{2}\right|^{2}+\left(f\left(u_{1}+u_{2}\right)-f\left(u_{1}\right), v_{2}\right) \\
& \quad \leq\left|g-g_{\epsilon} \| v_{2}\right| .
\end{aligned}
$$

For the last term on the left-hand side of (3.13), by (2.3), Proposition 2.1, and (3.7), there exists $\xi$ such that

$$
\begin{aligned}
\left(f\left(u_{1}+u_{2}\right)-f\left(u_{1}\right), v_{2}\right) & =\left(f^{\prime}\left(u_{1}+\xi u_{2}\right) u_{2}, v_{2}\right) \\
& \geq-c_{2}\left(1+\left|u_{1}\right|^{p}+\left|u_{2}\right|^{p}\right)\left|u_{2}\right|\left|v_{2}\right| \\
& \geq-c_{11}\left(M_{0}, M_{1}\right)\left|u_{2}\right|\left|v_{2}\right| .
\end{aligned}
$$

By the Gronwall and Poincaré inequalities,

$$
\begin{aligned}
\left\|\varphi^{2}\right\|_{E}^{2} & \leq \frac{2\left|g-g_{\epsilon}\right|^{2}}{\left(\frac{2 \sqrt{\lambda}}{\sqrt{\mu}}+c_{11}\left(M_{0}, M_{1}\right)-\sigma\right)\left(\alpha \lambda+\beta_{1}\right)} \\
& \leq \frac{8 \epsilon^{2}}{\left(\frac{2 \sqrt{\lambda}}{\sqrt{\mu}}+c_{11}\left(M_{0}, M_{1}\right)-\sigma\right)\left(\alpha \lambda+\beta_{1}\right)} .
\end{aligned}
$$

By (3.12), (3.15), and the following lemma we see that $\left\{S_{k}^{1}(t) \mid t \geq 0\right\}$ is compact in $\dot{H}^{1} \times L^{2}$. 
Lemma 3.1 (see [25]) Let $X$ be a complete metric space and $A$ be a subset in $X$, such that

$$
\forall \epsilon, \quad A \subset K_{\epsilon}+B(0, C(\epsilon))
$$

with $\lim _{\epsilon \rightarrow 0} C(\epsilon)=0$ and $K_{\epsilon}$ is compact in $X$, then $A$ is compact in $X$.

Third step: Let $\varphi^{3}=\left(u^{3}, v^{3}\right)$, by (3.4) and Lemma 2.2, we have

$$
\frac{1}{2} \frac{d}{d t}\left\|\varphi^{3}\right\|_{E}^{2}+\sigma\left\|\varphi^{3}\right\|_{E}^{2}+\frac{\alpha \lambda+\beta_{1}}{2}\left|v_{3}\right|^{2} \leq-\left(f(u)-f\left(u_{1}+u_{2}\right), v_{3}\right)+\lambda\left(u_{3}, v_{3}\right) .
$$

For the right-hand side of (3.16), by (2.1), we have

$$
\begin{aligned}
-\left(f(u)-f\left(u_{1}+u_{2}\right), v_{3}\right)+\lambda\left(u_{3}, v_{3}\right) & \leq c_{13}\left|u_{3} \| v_{3}\right|+\lambda\left|u_{3}\right|\left|v_{3}\right| \\
& \leq c_{14}\left\|u_{3}\right\|\left|v_{3}\right| \\
& \leq \frac{\sigma}{2}\left\|\varphi^{3}\right\|_{E}^{2}+\left(\frac{c_{14}^{2}}{2 \sigma \mu}-\frac{\sigma}{2}\right)\left|v_{3}\right|^{2} .
\end{aligned}
$$

Let $c_{14}<\sqrt{\sigma \mu\left(\alpha \lambda+\beta_{1}\right)+\sigma^{2} \mu}$. Equations (3.16) and (3.17) lead to

$$
\left\|\varphi^{3}\right\|_{E}^{2} \leq\left(\mu\left\|u_{0}\right\|^{2}+\left|v_{0}\right|^{2}\right) \exp \{-\sigma t\}
$$

In other words we have

$$
S(t)\left(u_{0}, v_{0}\right) \rightarrow 0 \quad \text { in } \dot{H}^{1} \times L^{2},
$$

uniformly in bounded sets. Then from Theorem 3.1, (3.18), and the compactness of $\left\{S_{k}^{1}(t) \mid\right.$ $t \geq 0$, for the system (1.1) there exists a global attractor $\mathcal{A}$ in $\dot{H}^{1} \times L^{2}$.

Remark 3.2 It is easy to see that the semigroup

$$
S_{k}(t)=S_{k}^{1}(t)+S_{k}^{2}(t):\left(u_{0}, v_{0}=u_{1}+k u_{0}\right)^{T} \rightarrow\left(u(t), u_{t}(t)+k u(t)\right)^{T}, \quad E \rightarrow E,
$$

defined by (2.12) has the following relation with $S(t)$ :

$$
S_{k}(t)=R_{k} S(t) R_{-k},
$$

where $R_{k}$ is an isomorphism of $E$ :

$$
R_{k}:\left\{u, u_{t}\right\} \rightarrow\left\{u, u_{t}+k u\right\}
$$

Since the semigroup $\left\{S_{k}(t) \mid t \geq 0\right\}$ defined by (1.1) possesses a global attractor $\mathcal{A}_{0} \subset E$, by (3.19), $\{S(t) \mid t \geq 0\}$ also possesses a global attractor $\mathcal{A}=R_{k} \mathcal{A}_{0}$.

\section{Regularity of the attractor}

In this section, we suppose that $g \in \dot{L}^{2}$ and prove that the attractor is compact in $H^{2} \times H^{1}$. Let $\left(u_{0}, v_{0}\right) \in \dot{H}^{1} \times L^{2}$. We set

$$
S_{k}(t)\left(u_{0}, v_{0}\right)=(p(t), q(t))+(w(t), \rho(t)), \quad \forall t \geq 0,
$$


where $(p, q)$ is the solution of

$$
\left\{\begin{array}{l}
p_{t}+k p-q=0 \\
q_{t}+A_{1} p-k\left(\alpha A_{1}-k\right) p+\left(\alpha A_{1}-k\right) q+h_{1}(q-k p)+f(u)-\lambda u=g \\
p(0)=0, \quad q(0)=0
\end{array}\right.
$$

$(w, \rho)$ satisfies

$$
\left\{\begin{array}{l}
w_{t}+k w-\rho=0 \\
\rho_{t}+A_{1} w-k\left(\alpha A_{1}-k\right) w+\left(\alpha A_{1}-k\right) \rho+h_{1}(v-k u)-h_{1}(q-k p)=0 \\
w(0)=u_{0}, \quad \rho(0)=v_{0} .
\end{array}\right.
$$

We use Remark 3.1 to prove that $\mathcal{A} \subset H^{2} \times H^{1}$. Let $\left(u_{0}, v_{0}\right) \in \mathcal{A}$. Let $\left(u_{0}^{n}, v_{0}^{n}\right) \in \mathcal{B}$ and a sequence of a real numbers $t_{n} \rightarrow \infty$ as $n \rightarrow \infty$, such that

$$
S_{k}\left(t_{n}\right)\left(u_{0}^{n}, v_{0}^{n}\right) \rightarrow\left(u_{0}, v_{0}\right) \quad \text { in } \dot{H}^{1} \times L^{2} \text { as } n \rightarrow \infty .
$$

We also have

$$
S_{k}\left(t_{n}\right)\left(u_{0}^{n}, v_{0}^{n}\right)=\left(p^{n}\left(t_{n}\right), q^{n}\left(t_{n}\right)\right)+\left(w^{n}\left(t_{n}\right), \rho^{n}\left(t_{n}\right)\right), \quad \forall n \in \mathbb{N} .
$$

We deduce from (3.12) and (3.20) that

$$
\left\|\left(p^{n}\left(t_{n}\right), q^{n}\left(t_{n}\right)\right)\right\|_{H^{2} \times H^{1}}^{2} \leq \frac{1}{\rho \varrho} C^{3}\left(\left|A_{1}^{\frac{1}{2}} g_{\epsilon}\right|, \lambda, \alpha, \delta, \kappa, k, \beta_{1}, \beta_{2}\right)
$$

and

$$
\left\|\left(w^{n}\left(t_{n}\right), \rho^{n}\left(t_{n}\right)\right)\right\|_{\dot{H}^{1} \times L^{2}}^{2} \leq\left\|\left(u_{0}, v_{0}\right)\right\|_{H^{1} \times L^{2}}^{2} \exp \left(-2 \sigma t_{n}\right) .
$$

From (4.6), we infer that there exist subsequences $t_{n^{\prime}}$ and $\left(u_{0}^{n^{\prime}}, v_{0}^{n^{\prime}}\right)$, and $\left(p^{0}, q^{0}\right) \in H^{2} \times H^{1}$ such that

$$
\left(p^{n^{\prime}}\left(t_{n^{\prime}}\right), q^{n^{\prime}}\left(t_{n^{\prime}}\right)\right) \rightarrow\left(p^{0}, q^{0}\right) \quad \text { weakly in } H^{2} \times H^{1} .
$$

From (4.4), (4.5), and (4.7), we have

$$
\left(p^{n^{\prime}}\left(t_{n^{\prime}}\right), q^{n^{\prime}}\left(t_{n^{\prime}}\right)\right) \rightarrow\left(u_{0}, v_{0}\right) \quad \text { in } H^{1} \times L^{2} .
$$

We conclude that $\left(u_{0}, v_{0}\right)=\left(p^{0}, q^{0}\right)$ and then

$$
\mathcal{A} \subset H^{2} \times H^{1}
$$

In the following, we use the famous argument of [5] to show that the attractor $\mathcal{A}$ is actually a compact set in $H^{2} \times H^{1}$.

Theorem 4.1 The semigroup $\{S(t) \mid t \geq 0\}$ in $\dot{H}^{1} \times L^{2}$ possesses a global attractor $\mathcal{A}$ which is compact subset of $H^{2} \times H^{1}$. 
Proof Multiplying (1.1) by $\left(A_{1} u, A_{1} v\right)$, we have

$$
\begin{aligned}
& \frac{1}{2} \frac{d}{d t}\left[\mu\left\|A_{1}^{\frac{1}{2}} u\right\|^{2}+\left|A_{1}^{\frac{1}{2}} v\right|^{2}\right]+\sigma\left(\mu\left\|A_{1}^{\frac{1}{2}} u\right\|^{2}+\left|A_{1}^{\frac{1}{2}} v\right|^{2}\right)+\frac{\alpha \lambda+\beta_{1}}{2}\left|A_{1}^{\frac{1}{2}} v\right|^{2} \\
& \quad=\left(g, A_{1} v\right)-\left(f(u)-\lambda u, A_{1} v\right) .
\end{aligned}
$$

We put $G(u, v)=\mu\left\|A_{1}^{\frac{1}{2}} u\right\|^{2}+\left|A_{1}^{\frac{1}{2}} v\right|^{2}, K(u, v)=\int_{\Omega}\left[A_{1}^{\frac{1}{2}} g \cdot A_{1}^{\frac{1}{2}} v-(f(u)-\lambda u) A_{1} v\right] d x-$ $\frac{\alpha \lambda+\beta_{1}}{2}\left|A_{1}^{\frac{1}{2}} v\right|^{2}$, then we have

$$
\frac{1}{2} \frac{d}{d t} G(u, v)+\sigma G(u, v)=K(u, v)
$$

this shows that

$$
G(u, v)=e^{-2 \sigma t} G\left(u_{0}, v_{0}\right)+2 \int_{0}^{t} e^{2 \sigma(s-t)} K(u, v) d s .
$$

We consider a sequence $\left(a_{n}, b_{n}\right)_{n \in \mathbb{N}} \in \mathcal{A}$, and we may assume that, up to a subsequence,

$$
\begin{aligned}
& \left(a_{n}, b_{n}\right) \rightarrow(a, b) \text { weakly in } H^{2} \times H^{1}, \\
& \left(a_{n}, b_{n}\right) \rightarrow(a, b) \quad \text { strongly in } H^{1} \times L^{2} .
\end{aligned}
$$

We want to prove the strong convergence of $\left(a_{n}, b_{n}\right)$ in $H^{2} \times H^{1}$; this will give the compactness of $\mathcal{A}$ in $H^{2} \times H^{1}$.

For a given $T \geq 0$ and up to subsequence extraction, we may assume that a.e. in $t \in[0, T]$,

$$
\begin{aligned}
& S(t-T)\left(a_{n}, b_{n}\right) \rightarrow S(t-T)(a, b) \quad \text { weakly in } H^{2} \times H^{1}, \\
& S(t-T)\left(a_{n}, b_{n}\right) \rightarrow S(t-T)(a, b) \quad \text { strongly in } H^{1} \times L^{2} .
\end{aligned}
$$

Using (4.13) for $\left(u_{0}, v_{0}\right)=S(-T)\left(a_{n}, b_{n}\right)$, we have

$$
\begin{aligned}
G\left(S(t-T)\left(a_{n}, b_{n}\right)\right)= & e^{-2 \sigma t} G\left(S(-T)\left(a_{n}, b_{n}\right)\right) \\
& +2 \int_{0}^{t} e^{2 \sigma(s-t)} K\left(S(s-T)\left(a_{n}, b_{n}\right)\right) d s .
\end{aligned}
$$

By the Lebesgue dominated convergence theorem, similar to (3.8)-(3.12), we have

$$
\int_{0}^{t} e^{2 \sigma(s-t)} K\left(S(s-T)\left(a_{n}, b_{n}\right)\right) d s \rightarrow \int_{0}^{t} e^{2 \sigma(s-t)} K(S(s-T)(a, b)) d s,
$$

passing to the limit sup in (4.14) and taking $t=T$ we have

$$
\begin{aligned}
\varlimsup_{n \rightarrow \infty} G\left(a_{n}, b_{n}\right) \leq & \varlimsup_{n \rightarrow \infty} e^{-2 \sigma T} G\left(S(-T)\left(a_{n}, b_{n}\right)\right) \\
& +2 \int_{0}^{T} e^{2 \sigma(s-T)} K(S(s-T)(a, b)) d s .
\end{aligned}
$$


The last term of (4.16) follows from (4.14) for $\left(u_{0}, v_{0}\right)=S(-T)(a, b)$ and $t=T$, and we have

$$
I=2 \int_{0}^{T} e^{2 \sigma(s-T)} K(S(s-T)(a, b)) d s=G(a, b)-e^{-2 \sigma T} G(S(-T)(a, b)) .
$$

We replace $I$ in (4.16), we obtain

$$
\varlimsup_{n \rightarrow \infty} G\left(a_{n}, b_{n}\right) \leq G(a, b)-e^{-2 \sigma T}\left(G(S(-T)(a, b))-\varlimsup_{n \rightarrow \infty} G\left(S(-T)\left(a_{n}, b_{n}\right)\right)\right) .
$$

It is a standard matter to prove

$$
e^{-2 \sigma T}\left(G(S(-T)(a, b))-\varlimsup_{n \rightarrow \infty} G\left(S(-T)\left(a_{n}, b_{n}\right)\right)\right) \rightarrow 0 \quad \text { when } T \rightarrow \infty
$$

It follows from (4.18) that

$$
\varlimsup_{n \rightarrow \infty} G\left(a_{n}, b_{n}\right) \leq G(a, b) \leq \underline{\lim }_{n \rightarrow \infty} G\left(a_{n}, b_{n}\right) .
$$

This completes the proof of lemma.

\section{Competing interests}

The author declares to have no competing interests.

\section{Acknowledgements}

This work is supported by the National Natural Science Foundation of China (11101265) and Shanghai Education Research and Innovation Key Project (14ZZ157).

Received: 14 March 2014 Accepted: 9 September 2014 Published: 16 Oct 2014

\section{References}

1. Ghidaglia, JM, Marzocchi, A: Longtime behavior of strongly damped nonlinear wave equations, global attractors and their dimension. SIAM J. Math. Anal. 22, 879-895 (1991)

2. Landahl, PS, Soerensen, OH, Christiansen, PL: Soliton excitations in Josephson tunnel junctions. Phys. Rev. B 25 , 5337-5348 (1982)

3. Arrietta, J, Carvalho, AN, Hale, JK: A damped hyperbolic equations with critical exponents. Commun. Partial Differ. Equ. 17, 841-866 (1992)

4. Babin, AV, Vishik, MI: Attractors of Evolution Equations. North-Holland, Amsterdam (1992)

5. Ball, J: Global attractors for damped semilinear wave equations. Discrete Contin. Dyn. Syst. 10, 31-52 (2004)

6. Chepyzhov, WV Vishik, Ml: Attractor of Equations of Mathematical Physics. Am. Math. Soc., Providence (2002)

7. Eden, A, Milani, AJ, Nicolaenko, B: Finite-dimensional exponential attractors for semilinear wave equations with damping. J. Math. Anal. Appl. 169, 408-419 (1992)

8. Hale, JK: Asymptotic Behavior of Dissipative Systems. Am. Math. Soc., Providence (1988)

9. Ladyzhenskaya, OA: Attractors for Semigroups and Evolution Equations. Cambridge University Press, Cambridge (1991)

10. Temam, R: Infinite-Dimensional Dynamical Systems in Mechanics and Physics, 2nd edn. Springer, New York (1997)

11. Chueshov, I, Lasiecka, I: Attractors for second-order evolution equations with nonlinear damping. J. Dyn. Differ. Equ. $16,469-512(2004)$

12. Chueshov, I, Lasiecka, I: Long-time dynamics of a semilinear wave equation with nonlinear interior/boundary damping and sources of critical exponents. In: Control Methods in PDE-Dynamical Systems. Contemporary Mathematics. Am. Math. Soc., Providence (2007)

13. Feireisl, E: Global attractors for damped wave equations with supercritical exponent. J. Differ. Equ. 116, $431-447$ (1995)

14. Pata, V, Zelik, S: A remark on the damped wave equation. Commun. Pure Appl. Anal. 5, 611-616 (2006)

15. Pazy, A: Semigroups of Linear Operators and Applications to Partial Differential Equations. Appl. Math. Sci., vol. 44. Springer, New York (1983)

16. Sun, $\mathrm{CY}$, Yang, $\mathrm{MH}$, Zhong, CK: Global attractors for the wave equation with nonlinear damping. J. Differ. Equ. 227 427-443 (2006)

17. Sun, CY, Cao, DM, Duan, JQ: Uniform attractors for nonautonomous wave equations with nonlinear damping. SIAM J. Appl. Dyn. Syst. 6, 293-318 (2007)

18. Zhou, S: Dimension of the global attractor for damped nonlinear wave equations. Proc. Am. Math. Soc. 12, $3623-3631$ (1999) 
19. Li, HY, Zhou, S, Yin, FQ: Global periodic attractor for strongly damped wave equations with time-periodic driving force. J. Math. Phys. 45, 3462-3467 (2004)

20. Massatt, P: Limiting behavior for strongly damped nonlinear wave equations. J. Differ. Equ. 48, 334-349 (1983)

21. Pata, V, Zelik, S: Smooth attractors for strongly damped wave equations. Nonlinearity 19, 1495-1506 (2006)

22. Webb, GF: Existence and asymptotic behavior for strongly damped nonlinear wave equations. Can. J. Math. 32, 631-643 (1980)

23. Zhou, S: Global attractor for strongly damped nonlinear wave equations. Funct. Differ. Equ. 6, 451-470 (1999)

24. Caraballo, T, Carvalho, AN, Langa, JA, Rivero, F: A non-autonomous strongly damped wave equation: existence and continuity of the pullback attractor. Nonlinear Anal. 74, 2272-2283 (2011)

25. Atlas, A: Regularity of the attractor for symmetric regularized wave equation. Commun. Pure Appl. Anal. 4, 695-704 (2005)

10.1186/1029-242X-2014-396

Cite this article as: Li: Regularity of the attractor for strongly damped wave equations with nonlinearity. Journal of Inequalities and Applications 2014, 2014:396

Submit your manuscript to a SpringerOpen ${ }^{\circ}$ journal and benefit from:

- Convenient online submission

- Rigorous peer review

- Immediate publication on acceptance

- Open access: articles freely available online

- High visibility within the field

- Retaining the copyright to your article

Submit your next manuscript at $>$ springeropen.com 